\title{
Cortical and Subcortical Grey Matter Abnormalities in White Matter Hyperintensities and Subsequent Cognitive Impairment
}

\author{
Wenhao Zhu ${ }^{1}$ - Hao Huang ${ }^{1}$ - Shiqi Yang ${ }^{2}$ Xiang Luo ${ }^{1}$ Wenzhen $\mathrm{Zhu}^{2}$ • \\ Shabei $\mathrm{Xu}^{1} \cdot$ Qi Meng ${ }^{1}$ Chengchao Zuo ${ }^{1}$ - Yong Liu $^{3,4,5}$ - Wei Wang ${ }^{1}$. \\ Alzheimer's Disease Neuroimaging Initiative
}

Received: 21 July 2020/Accepted: 28 October 2020/Published online: 7 April 2021

(C) The Author(s) 2021

\begin{abstract}
Grey matter (GM) alterations may contribute to cognitive decline in individuals with white matter hyperintensities (WMH) but no consensus has yet emerged. Here, we investigated cortical thickness and grey matter volume in $23 \mathrm{WMH}$ patients with mild cognitive impairment (WMH-MCI), $43 \mathrm{WMH}$ patients without cognitive impairment, and 55 healthy controls. Both WMH groups showed GM atrophy in the bilateral thalamus, frontoinsular cortices, and several parietal-temporal regions, and the WMH-MCI group showed more extensive and severe GM atrophy. The GM atrophy in the thalamus and frontoinsular cortices was associated with cognitive decline in the
\end{abstract}

Wenhao Zhu and Hao Huang contributed equally to this work.

Supplementary Information The online version contains supplementary material available at https://doi.org/10.1007/s12264-02100657-0.

Yong Liu

yongliu@bupt.edu.cn

$\triangle$ Wei Wang

wwang@tjh.tjmu.edu.cn

1 Department of Neurology, Tongji Hospital, Tongji Medical College, Huazhong University of Science and Technology, Wuhan 430030, China

2 Department of Radiology, Tongji Hospital, Tongji Medical College, Huazhong University of Science and Technology, Wuhan 430030, China

3 Brainnetome Center and National Laboratory of Pattern Recognition, Institute of Automation, Chinese Academy of Sciences, Beijing 100190, China

4 School of Artificial Intelligence, Beijing University of Posts and Telecommunications, Beijing 100876, China

5 University of the Chinese Academy of Sciences, Beijing 100049, China
WMH-MCI patients and may mediate the relationship between WMH and cognition in WMH patients. Furthermore, the main results were well replicated in an independent dataset from the Alzheimer's Disease Neuroimaging Initiative database and in other control analyses. These comprehensive results provide robust evidence of specific GM alterations underlying WMH and subsequent cognitive impairment.

Keywords White matter hyperintensities - Cognitive impairment · Cortical thickness · Grey matter volume . Replication

\section{Introduction}

White matter hyperintensities (WMHs) are areas of high signals on fluid-attenuated inversion recovery (FLAIR) and T2-weighted MR images and are commonly observed in the brains of elderly individuals [1]. Converging evidence suggests that WMHs are involved in cognitive impairment in various populations, including normal aging, vascular dementia, and Alzheimer's disease (AD) [1,2]. Given that $\mathrm{WMH}$ is generally considered to represent the primary damage of cerebral small vessel disease (CSVD) due to chronic subcortical ischemia mainly involving subcortical white matter (WM) [1], previous studies concentrated on the role of WM disconnections, including aberrant WM microstructural integrity and networks in WMH progression and the cognitive decline of WMH patients [3-5]. Nevertheless, the relationship between WMH and cognitive impairment cannot be fully explained by WMH load or WM disruption, and it is still difficult to determine whether and to what extent cognitive decline exists in individual patients with WMH [1]. 
The grey matter (GM) is thought to play a key role in information extraction, exchange, and integration in cognitive processes. Recently, evidence has accumulated that morphological changes of GM are partly responsible for the cognitive decline in patients with WMH [6]. A few case-control studies have reported abnormalities of GM volume (GMV) or cortical thickness in subcortical vascular dementia [7-10] and subcortical vascular mild cognitive impairment (MCI) [10-13] which were mainly attributed to WMH, compared with healthy controls (HCs). Furthermore, the results of cross-sectional studies revealed that GMV (frontal or temporal cortices) mediates the relationship between WMH and cognition in non-demented individuals [14] and in a mixed sample of individuals with transient ischemic attacks, symptomatic coronary artery disease, and AD [15]. Another two cross-sectional studies showed that region-specific or global damage of cortical thickness mediates the relationship between WMH volume and cognition in CSVD [16] and older adults without dementia [17]. Overall, the results of these studies noted that specific cortical atrophy (especially GM atrophy in some frontal and temporal cortices) may be involved in the cognitive decline in WMH patients. However, the results of these studies show great divergences and there is no consensus on the characteristic structural alterations in GM underlying WMH and subsequent cognitive impairment.

The inconsistency among the few previous studies may be heterogeneity inherent to study design, different GM measurements, and various pipelines of data pre-processing and analysis. In the case-control studies that focused on GM changes in WMH patients, morphological comparisons were conducted mainly between those with cognitive impairment and $\mathrm{HCs}$, and there was no information on the GM characteristics of WMH patients with normal cognition. Meanwhile, the heterogeneity of the population (i.e., a mixed sample with $\mathrm{AD}$ or participants with other diseases [15], different race and ethnicity, and all degrees of WMH $[14,17])$ may also lead to inconsistency. These paradigms impede the exploration of a comprehensive picture of GM alterations along with the progression of $\mathrm{WMH}$ and subsequent cognitive impairment. In addition, it should also be noted that these studies performed GM morphometric analyses at different levels (the regional level [9, 13-17] or the voxel level [7, 8, 10-12]), using disparate methodological steps in data pre-processing and statistical analysis, which makes it difficult to directly compare the results across different studies.

In this study, we aimed to evaluate the GM structural alterations underlying cognitive decline in $\mathrm{WMH}$ patients. The two conventional GM measurements cortical thickness and GMV provide similar, but sometimes relatively unique, information on different types of structural changes [18-21]. And compared to the voxel-based method, GM morphometric analyses at the regional level provide direct and precise information of GM alteration in each subregion $[22,23]$. Hence, we explored the role of GM alterations in WMH and subsequent cognitive impairment through studying whole-brain alteration patterns of both cortical thickness and GMV among WMH patients with MCI (WMH-MCI), WMH patients with no cognitive impairment (WMH-nCI), and healthy controls (HCs) using the fine-grained Human Brainnetome Atlas [24]. Importantly, we further conducted a series of verification analyses, including conducting GM morphometric analyses across the three groups in the ADNI database, at the voxel level, using different brain atlases, and performing group classifications, to validate the reproducibility and generalizability of the main results.

\section{Materials and Methods}

All participants included in the in-house dataset were recruited from the outpatient service of the Department of Neurology, Tongji Hospital, between 2014 and 2016. The study was approved by the Ethics Committee of Tongji Hospital, Tongji Medical College, Huazhong University of Science and Technology (ID: TJ-C20131216). Written informed consent was given by each participant. Here we only provide a brief introduction to the participants; the details can be found in our previous study [25].

\section{Participants}

One hundred and forty right-handed individuals, including 80 consecutive patients with moderate to severe WMH (defined as the sum of the deep WMH Fazekas score and the periventricular WMH Fazekas score $\geq 3$ on FLAIR sequence images) $[26,27]$ and $60 \mathrm{HCs}$ without moderate to severe $\mathrm{WMH}$ and lacunes were initially recruited. All participants underwent clinical interviews and a battery of neuropsychological tests, including the Mini-Mental State Examination (MMSE), the clinical dementia rating (CDR), the Trail Making Test (TMT), the Symbol Digit Modalities Test (SDMT), the Digit Span Test (DST), the Verbal Fluency Test (VFT), the Auditory Verbal Learning Test (AVLT), and the Hamilton depression rating scale (24items). The neuropsychological tests were composited into three cognitive domains [28]: processing speed (TMT-A and SDMT) [29], executive function (DST backward, VFT, and TMT-B) [30], and memory (AVLT immediate recall, short delay recall, long delay recall, and long delay recognition) [31] (The details of compounded scores for each domain are shown in the Supplementary material).

As in our previous study [25], the inclusion criteria for WMH patients were: (1) moderate to severe WMH with/ 
without lacunes; (2) no dementia based on the criteria of the Diagnostic and Statistical Manual of Mental Disorders, Fourth Edition; a CDR score $\leq 0.5$; an MMSE score $\geq 20$ (primary school) or $\geq 24$ (junior school or above); and the ability to perform all activities of daily living independently [32]. The HCs had no history of neuropsychiatric disorders and no cognitive complaints.

The exclusion criteria for participants in this study included the following items: (1) left-handedness; (2) $<5$ years of education; (3) the presence of any infarct with a diameter $>20 \mathrm{~mm}$ on T1-weighted images, cortical infarcts, or cerebral hemorrhage; (4) WMH mimics (e.g., multiple sclerosis, hypoxic-ischemic encephalopathy, and leukodystrophy); (5) a history of Parkinson's disease, epilepsy or psychiatric diseases; (6) use of medications that may affect cognitive function; (7) insufficient cooperation with the study procedures for any reason; and (8) a Hamilton depression scale score (24 items) $\geq 20$. Sixtyeight WMH patients and $56 \mathrm{HCs}$ with completed neuropsychological tests and MR scans fulfilled the above criteria. Two WMH participants with excessive head motion ( $>3 \mathrm{~mm}$ translation or $>3^{\circ}$ angular rotation on any axis) during scanning and one $\mathrm{HC}$ without a complete set of MRI data due to technical problems were excluded.

Based on cognitive ability, we then divided the WMH patients into two groups: (1) the WMH-MCI group, which included patients with a score $\geq 0.5$ for at least one of the CDR domains, and objective evidence that one or more cognitive domains were impaired (age- and educationadjusted z-scores at least $1.5 \mathrm{SD}$ below those of the HCs on one or more cognitive domains) [28, 32]; and (2) the WMH-nCI group, which included patients with a score of 0 on all six CDR domains, an MMSE score $\geq 24$ (primary school) or $\geq 27$ (junior school or above) and no objective evidence of impairment in any of the three cognitive domains. No participants were excluded by this procedure. Finally, a total of 121 participants (23 WMH-MCI, 43 WMH-nCI, and $55 \mathrm{HC}$ ) were included in the in-house dataset.

\section{MRI Acquisition}

MR images were acquired on a 3.0T MR scanner (Discovery MR750, GE Healthcare, Milwaukee, WI, USA) using a 32-channel head array coil and included whole-brain T1-weighted, T2-weighted, fluid-attenuated inversion recovery (FLAIR), and a BRAin Volume (BRAVO) sequence. FLAIR images were collected using a repetition time $(\mathrm{TR})=8,000 \mathrm{~ms}$, echo time $(\mathrm{TE})=160 \mathrm{~ms}$, inversion time $(\mathrm{TI})=2,100 \mathrm{~ms}$, flip angle $(\mathrm{FA})=111^{\circ}, \quad$ slice $\quad$ thickness $=5.0 \mathrm{~mm}$, slice gap $=1.5 \mathrm{~mm}$, data matrix $=512 \times 512$, and field of view $\quad(\mathrm{FOV})=240 \times 240 \mathrm{~mm}^{2} . \quad$ High-resolution anatomical T1-weighted images were obtained using a sagittal BRAVO sequence with $\mathrm{TR}=8.16 \mathrm{~ms}$, $\mathrm{TE}=3.18 \mathrm{~ms}, \quad \mathrm{TI}=50 \mathrm{~ms}, \quad \mathrm{FA}=12^{\circ}, \quad$ number of slices $=188, \quad$ slice $\quad$ thickness $=1.0 \mathrm{~mm}, \quad$ data matrix $=256 \times 256, \mathrm{FOV}=256 \times 256 \mathrm{~mm}^{2}$, and voxel size $=1 \mathrm{~mm} \times 1 \mathrm{~mm} \times 1 \mathrm{~mm}$.

\section{MRI Data Processing}

We used the CAT12 toolbox (Computational Anatomy Toolbox; http://www.neuro.uni-jena.de/cat) implemented in SPM12 (Statistical Parametric Mapping, Institute of Neurology, London, UK) for voxel-based morphometry (VBM) and surface-based morphometry (for cortical thickness) analysis of imaging data [33-35]. Before pre-processing, all images were visually checked for the same orientation. Then they were corrected for bias-field inhomogeneities, aligned with the International Consortium for Brain Mapping template, and normalized using the DARTEL algorithm. The images were then segmented into GM, WM, and cerebrospinal fluid according to the tissue probability map provided in the CAT12 toolbox with voxel size $=1 \mathrm{~mm}$ $1 \mathrm{~mm} \times 1 \mathrm{~mm}$. Furthermore, the segmented GM was modulated by the nonlinear normalization parameters to correct for individual brain size. After the pre-processing pipeline was completed, all scans passed the automated quality check protocol included in the CAT12 toolbox. For VBM analysis, the modulated and warped GM was finally smoothed by convolution with an isotropic Gaussian kernel, 6-mm full-width at half-maximum (FWHM) and total intracranial volume (TIV) was also calculated.

Cortical thickness analysis was implemented by the fully automated projection-based thickness method in the CAT12 toolbox. The algorithms uses tissue segmentation to estimate $\mathrm{WN}$ distance and project the local maxima to other GM voxels using the neighbor relationship described by the WM distance [33]. The surface data were resampled to the Human Connectome Project 32K template and were smoothed with a 15-mm FWHM Gaussian kernel.

As in our previous study [25], two independent raters (H.H. and Q.M.), blinded to the clinical information, manually segmented WMH from FLAIR images using ITK-SNAP 3.6.0 (http://www.itksnap.org/pmwiki/) and calculated the WMH volume as the sum of the WMH of all the image layers (details described in our previous study).

\section{Statistical Analyses}

\section{Atlas-Based Analysis}

To characterize the whole-brain GMV and cortical thickness changes of each participant, we used the Human 
Brainnetome Atlas [24], which consists of 246 subregions in the cerebrum (210 cortical and 36 subcortical) (http:// atlas.brainnetome.org). All 246 regions for GMV and 210 regions for cortical thickness were investigated. One-way analysis of variance (ANOVA) and post-hoc analysis were implemented to identify differences in GMV and cortical thickness among the three groups $(P<0.05$, Bonferroni corrected), controlling for age, gender, years of education, and TIV (for GMV) [36, 37] or age, gender, and years of education (for cortical thickness) [20] as confounding variables.

\section{Correlation Analyses and Mediation Analyses}

Differences in clinical characteristics, correlation analysis, and mediation analysis were calculated using SPSS 20.0 (IBM Corp., Armonk, NY, USA). Pearson's correlation analyses were used to evaluate the relationships between adjusted GM measures that were controlled for age, gender, and years of education (for GMV, added controlled for TIV) in all identified regions using the atlas-based approach and the z-scores of each cognitive domain in the WMH-MCI group $(P<0.05)$. Furthermore, to determine whether the relationship between WMH and early cognitive decline can be explained by regional GM changes in the WMH patients, exploratory mediation analyses were further constructed in these patients using the PROCESS macro in SPSS [38], with altered GM measures (cortical thickness and GMV) of identified regions by betweengroup analysis as mediators and cognitive z-scores as outcomes, controlling for age, sex, years of education, and TIV. To improve the normality of the WMH variable, the total WMH volume of each individual was log-transformed. The relationship between $\mathrm{WMH}$ volume and cognitive performance (total effect, path c), and the associations between WMH volume and GM measures (path a), were also determined in each mediation model. Mediation analyses were evaluated using a bootstrap method $(n=5,000)$, and significant indirect effects were defined by a $95 \%$ confidence interval entirely above or below 0 .

\section{Control Analyses}

\section{Voxel-Based Analysis}

To verify the results of GM abnormalities in WMH patients with and without cognitive impairment, complementary whole-brain voxel-wise analyses were also conducted among the three groups. The significant voxel-wise cortical thickness or GMV was determined using one-way ANOVA of the preprocessed imaging data among the three groups, with age, gender, years of education (adding TIV for GMV) as confounding variables. All analyses were restricted to the Brainnetome Atlas mask as the reference template, and the results were assessed at a peak threshold of $P<0.01$ (FDR corrected, cluster size $>70$ voxels).

\section{Replication of Main Results Using the ADNI Database}

To further validate the robustness of the main results, we also compared the differences in cortical thickness and GMV among the three groups in the replication dataset from the ADNI (ADNI-2 and ADNI-GO) cohort with 154 individuals: $62 \mathrm{WMH}-\mathrm{MCI}, 23 \mathrm{WMH}-\mathrm{nCI}$, and $69 \mathrm{HCs}$ (http://adni.loni.usc.edu; details in the supplementary material). The same quality-control criteria and pre-processing pipeline described above were applied to the replication dataset. The WMH quantification approach in the replication dataset is detailed on the ADNI site (http:// adni.loni.usc.edu) and in previous studies [39, 40]. To avoid the effect of correction for multiple comparisons and to evaluate the trend in whole-brain GM alterations among the three groups, correlation analyses between the in-house dataset and the replication dataset were calculated for each GM measure to explore whether the pattern of GM between-group differences was regionally consistent between the two datasets. For each GM measure, Pearson's correlation of F-scores of ANOVA between the two datasets was calculated at the regional level and the voxel level.

\section{Replication of Main Results from Different Perspectives}

To further verify the reproducibility of our main results, we repeated the between-group comparisons using different fine-grained brain atlases, including an atlas from the Human Connectome Project which consists of 360 regions [41], and two atlases of the Multiresolution Intrinsic Segmentation Template which consist of 325 regions and 444 regions [42]. We also repeated the analyses by adding hypertension and diabetes as covariates to investigate the potential confusion of the two risk factors which are considered to be involved in the progression of $\mathrm{WMH}$ underlying cognitive decline in WMH patients and verify the stability of our main results. In addition to the univariate analyses, we further conducted a group classification using logistic regression to explore whether the regional GM measures can be used as neuroimaging markers to discriminate WMH patients with and without early cognitive impairment. 
Table 1 Demographic and clinical features of the participants in the in-house dataset.

\begin{tabular}{|c|c|c|c|c|}
\hline & $\mathrm{HC}(n=55)$ & WMH-nCI $(n=43)$ & WMH-MCI $(n=23)$ & Overall $P$ Value \\
\hline \multicolumn{5}{|l|}{ Demographic data } \\
\hline Age (years) & $63.29 \pm 6.50$ & $65.72 \pm 5.94$ & $65.17 \pm 6.65$ & $0.089^{\mathrm{a}}$ \\
\hline Gender $(\mathrm{M} / \mathrm{F})$ & $32 / 23$ & $27 / 16$ & $15 / 8$ & $0.813^{\mathrm{b}}$ \\
\hline Education (years) & $10.75 \pm 3.29$ & $10.28 \pm 3.60$ & $9.34 \pm 3.89$ & $0.251^{\mathrm{a}}$ \\
\hline Hypertension, $n(\%)$ & $10(18.18 \%)$ & $31(72.09 \%)^{\mathrm{d}}$ & $15(62.22 \%) \mathrm{e}$ & $<0.001^{\mathrm{b}}$ \\
\hline Diabetes, $n(\%)$ & $3(5.45 \%)$ & $7(16.28 \%)^{\mathrm{d}}$ & $5(21.74 \%) \mathrm{e}$ & $<0.001^{\mathrm{b}}$ \\
\hline \multicolumn{5}{|l|}{ Structural MRI features } \\
\hline TIV $\left(\mathrm{cm}^{3}\right)$ & $1517.73 \pm 122.57$ & $1550.85 \pm 129.13$ & $1500.78 \pm 129.27$ & $0.248^{\mathrm{c}}$ \\
\hline Fazekas WMH score & $1.00 \pm 0.79$ & $4.47 \pm 1.18 \mathrm{~d}$ & $5.13 \pm 1.10 \mathrm{e}$ & $<0.001^{\mathrm{a}}$ \\
\hline Total WMH volume $\left(\mathrm{cm}^{3}\right)$ & $0.04 \pm 0.09$ & $13.53 \pm 13.97^{\mathrm{d}}$ & $18.19 \pm 15.02 \mathrm{e}$ & $<0.001^{\mathrm{a}}$ \\
\hline \multicolumn{5}{|l|}{ Cognitive performance } \\
\hline MMSE & $28.93 \pm 1.30$ & $28.77 \pm 1.23$ & $25.30 \pm 2.72^{\mathrm{e}, \mathrm{f}}$ & $<0.001^{\mathrm{a}}$ \\
\hline Processing speed ${ }^{\mathrm{g}}$ & $0.00 \pm 0.91$ & $0.04 \pm 0.82$ & $-1.53 \pm 0.95^{\mathrm{e}, \mathrm{f}}$ & $<0.001^{\mathrm{c}}$ \\
\hline Executive function ${ }^{\mathrm{g}}$ & $0.00 \pm 0.72$ & $-0.26 \pm 0.57$ & $-1.52 \pm 0.68^{\mathrm{e}, \mathrm{f}}$ & $<0.001^{\mathrm{c}}$ \\
\hline Memory $^{\mathrm{g}}$ & $0.00 \pm 0.85$ & $-0.25 \pm 0.87$ & $-1.71 \pm 0.63^{\mathrm{e}, \mathrm{f}}$ & $<0.001^{\mathrm{c}}$ \\
\hline
\end{tabular}

The data presented here are described in our previous study (Zhu et.al. 2019)

$T I V$ total intracranial volume, MMSE mini-mental state examination

${ }^{\mathrm{a}}$ Kruskal-Wallis test

${ }^{\mathrm{b}} \chi^{2}$ test

${ }^{\mathrm{c}}$ One-way analysis of variance

${ }^{\mathrm{d}}$ Significant difference between the WMH-nCI and HC groups

${ }^{\mathrm{e}}$ Significant difference between the WMH-MCI and $\mathrm{HC}$ groups

${ }^{\mathrm{f}}$ Significant difference between the WMH-MCI and WMH-nCI groups

${ }^{\mathrm{g}} \mathrm{Z}$-scores. The performance of processing speed, executive function, and memory is presented as the compound z-scores for the related tests with $\mathrm{HC}$ scores as reference

\section{Results}

\section{Participant Characteristics}

The demographic, neuroimaging, and cognitive characteristics are the same as our previous study [25] and are summarized in Table 1. Briefly, no significant differences were found in age, gender, education level, and TIV among the three groups. Both WMH-MCI and WMH-nCI patients exhibited higher WMH volumes and Fazekas scores than HCs, whereas the differences of WMH volume and Fazekas score were not significant between the WMH-MCI and WMH-nCI groups. The WMH-MCI patients showed significantly worse cognitive performance (MMSE and $z$-score of the three cognitive domains) than the other two groups, and there were no differences in cognitive function between the WMH-nCI and $\mathrm{HC}$ groups (details of raw scores in each neuropsychological test in Table S1).

\section{GM Alteration Patterns in WMH-MCI and WMH- nCI Patients}

At the regional level, significant GM alterations were distributed widely in cortical and subcortical regions among the three groups. Compared with HCs, both the WMH-MCI and the WMH-nCI patients showed a clear cortical GM atrophy pattern (the cortical thickness and GMV exhibited similar atrophy patterns) with predominant involvement of frontal, insular, parietal, and a few temporal regions, especially in the bilateral fronto-operculum/anterior insula, dorsolateral prefrontal cortex (DLPFC), and several regions of the inferior parietal lobe (IPL). More extensive and severe GM atrophy in these regions were identified in the WMH-MCI group relative to the WMH-nCI group. Subcortical nuclei were also affected in the WMH-MCI and the WMH-nCI patients. We found a significant reduction of GMV in the bilateral thalamus in the WMH-nCI patients compared with HCs, and nearly all subregions of the bilateral thalamus were more deeply involved in the WMH-MCI patients. Interestingly, relative 
to the HC group, the two WMH groups exhibited increased GMV in the bilateral caudate whereas no GM differences were identified between the WMH-MCI and WMH-nCI groups (Fig. 1; Tables S2, S3).

\section{Relationships Between GM Alteration, WMH, and Cognitive Performance}

In the WMH-MCI group, cortical thinning in most of the identified regions, mainly the bilateral fronto-operculum/ anterior insula, DLPFC, and IPL, were positively associated with processing speed and executive function (Fig. 2 and Supplementary Table S4). Meanwhile, we identified GMV-cognition relationships in several subregions of the thalamus (for processing speed and executive function), as well as a region in the left fronto-operculum (for processing speed) (Fig. 3 and Table S5).

In all WMH patients, there were significant associations between log-transformed WMH volume and processing speed $(r=-0.742, P<0.001)$ and executive function $(r=-0.509, P=0.003)$, and no correlation between logtransformed WMH volume and memory $(r=-0.311$, $P=0.152$ ). Significant associations were also found in the WMH patients between log-transformed WMH volume and GMV reduction in the thalamus, as well as reduction of GMV and cortical thickness in multiple identified frontal, insular, parietal, and a few temporal regions. Since no significant correlation was evaluated between WMH load and memory, mediation models were established only for processing speed and executive function. The mediation effects of WMH on cognition were identified via GM atrophy in several cortical and subcortical regions, mainly several regions of the thalamus (for GMV), and frontoinsular cortices (for cortical thickness and/or GMV). In addition, the mediation analyses indicated that specific GM atrophy mainly mediates the relationships between WMH and processing speed or executive function (Fig. 4 and Tables S6, S7).

\section{Control Results}

At the voxel level, the similar GM alteration maps (identified by GMV and cortical thickness) among the three groups were well replicated in the replication dataset from the ADNI database (details in Figs S1, S2 and Tables S8, S9). Briefly, the replication dataset from the ADNI database showed a very similar alteration patterns among the WMH-MCI, WMH-nCI, and HC groups in the demographic, neuroimaging, and cognitive metrics (Table S10). The patterns of GM abnormalities showed spatial consistency between the two datasets at either the regional level (cortical thickness, $r=0.524, P<0.001$; GMV, $r=0.522, P<0.001$ ) or the voxel level (cortical thickness, $\quad r=0.537, \quad P<0.001 ; \quad$ GMV,$\quad r=0.527$,

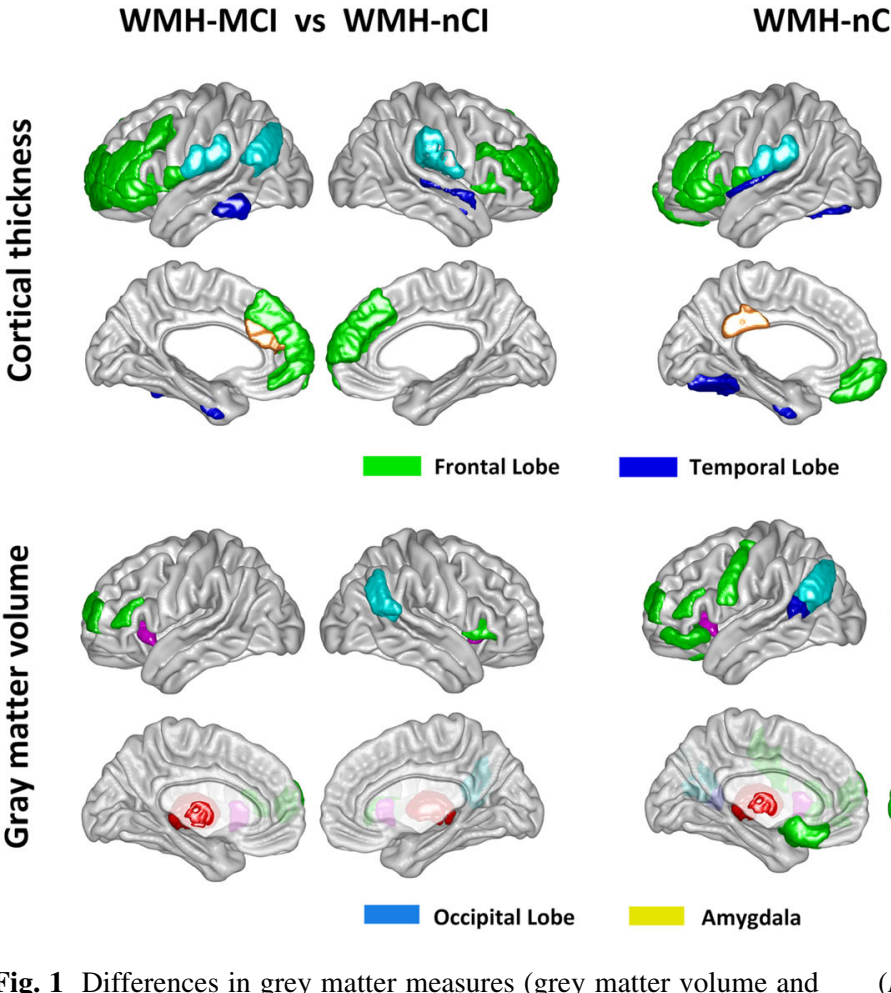

Fig. 1 Differences in grey matter measures (grey matter volume and cortical thickness) among the three groups in the in-house dataset based on the Human Brainnetome atlas with Bonferroni correction
$(P<0.05)$. The regions of altered cortical thickness or grey matter volume across the three groups are represented by different colors. 
A
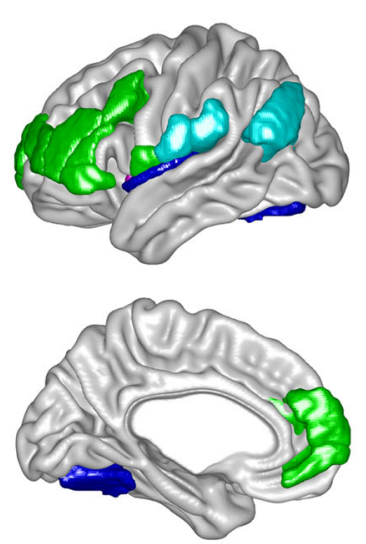

C

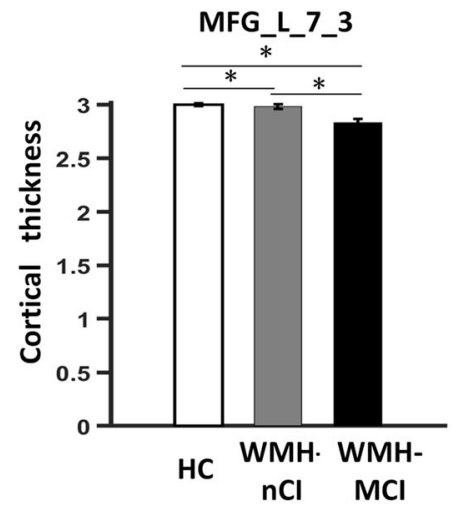

D

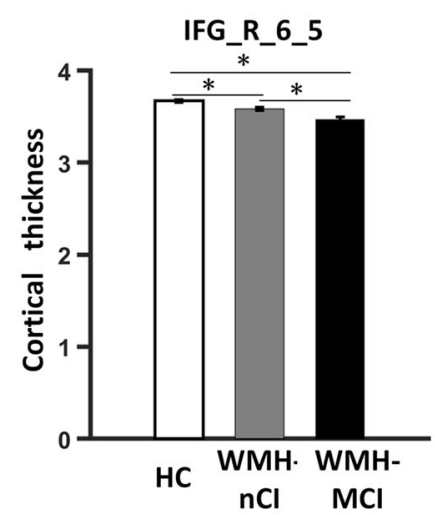

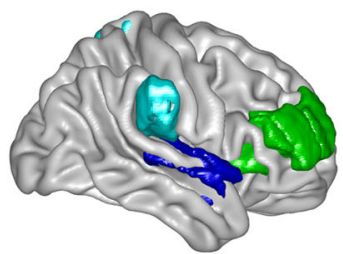

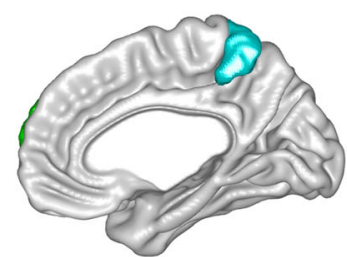

B
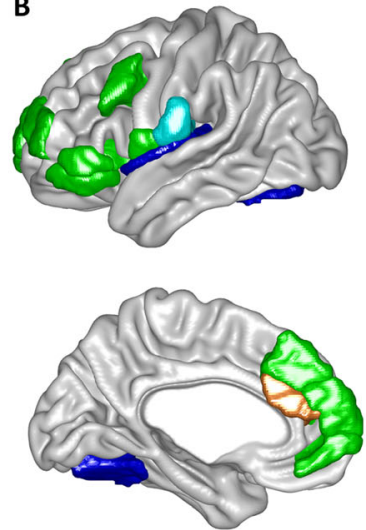

MFG_L_7_3

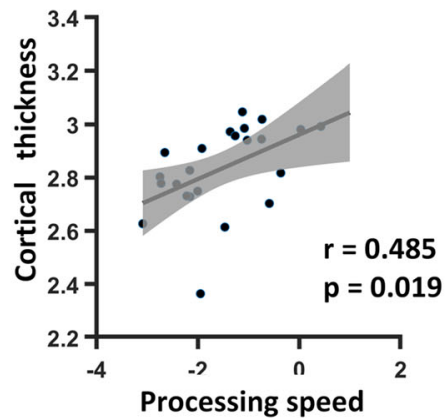

IFG_R_6_5

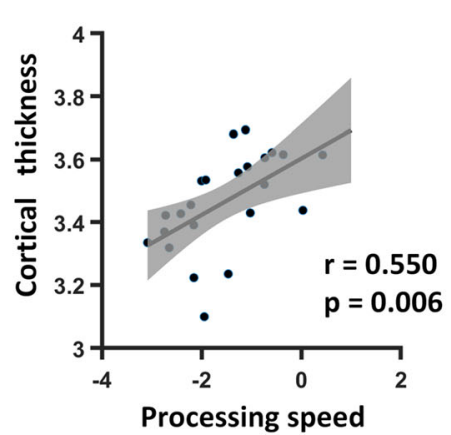

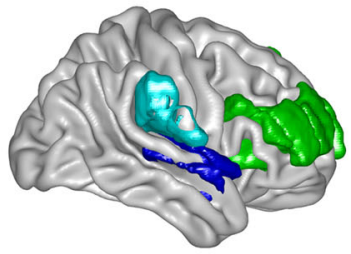

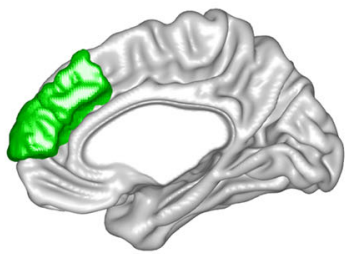

MFG_L_7_3

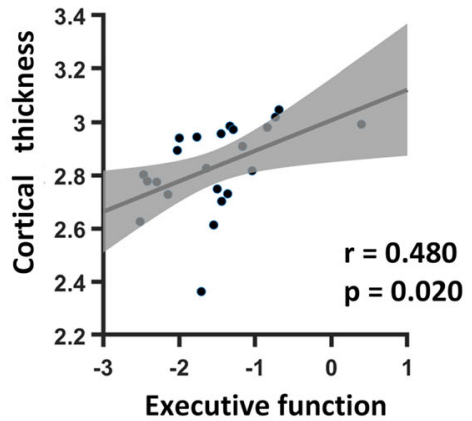

IFG_R_6_5

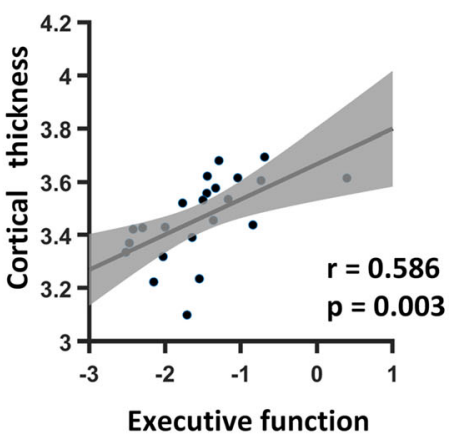

Fig. 2 A, B Regions of significant correlation between mean cortical thickness and cognitive performance [processing speed (A) and executive function $(\mathbf{B})$ ] in WMH-MCI patients $(P<0.05$, uncorrected). C, D Mean cortical thickness in representative regions of the fronto-operculum $(\mathbf{C})$ and DLPFC (D) in the three groups and scatter

$P<0.001$ ) (Fig. 5). The WMH-MCI and WMH-nCI groups of the replication dataset exhibited GM atrophy mainly in the bilateral thalamus, fronto-insular, temporal, and a few parietal regions compared with the HC group, and GM atrophy was more widespread and severe in the WMH-MCI group (Fig. S3 and Tables S11, S12).

As a control analysis for the choice of brain parcellation, we created a similar GM alteration map (revealed by the cortical thickness and GMV) among the three groups using plots of the association between the mean cortical thickness in these regions and cognitive performance in WMH-MCI patients. DLPFC, dorsolateral prefrontal cortex; IFG, inferior frontal gyrus; MFG, middle frontal gyrus; $\mathrm{L}$, left; $\mathrm{R}$, right. $* P<0.05$ (Bonferroni corrected).

the different brain atlases (Figs. S4, S5). Furthermore, we found significant correlations $(r=0.95, P<0.0001$ for cortical thickness; $r=0.97, \quad P<0.0001$ for GMV) between the F-score in the main result with and without controlling for hypertension and diabetes, emphasizing the robustness of our results and indicating that other risk factors such as hypertension and diabetes are unlikely to provide a plausible explanation of the GM alterations underlying cognitive decline in $\mathrm{WMH}$ patients revealed in 

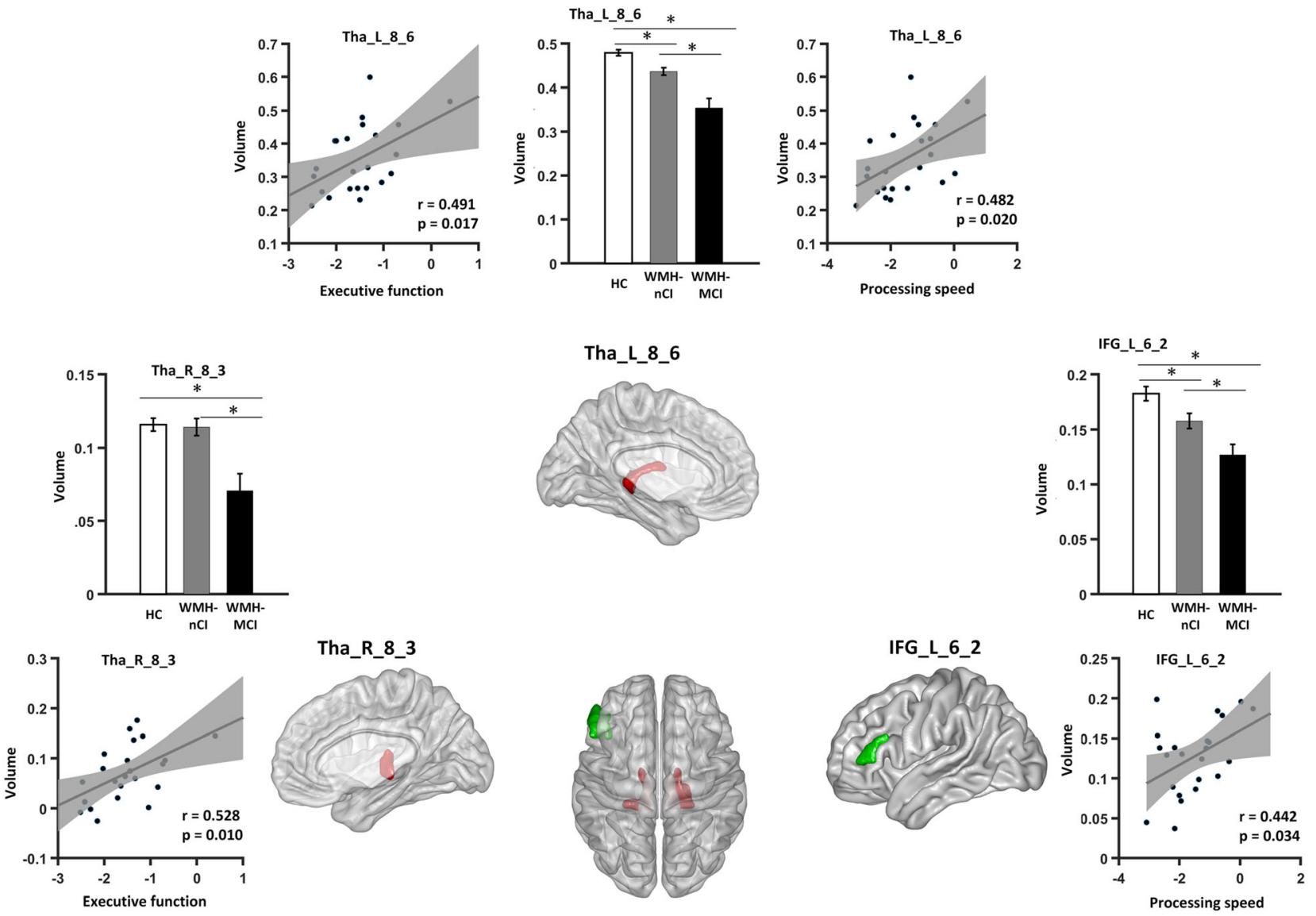

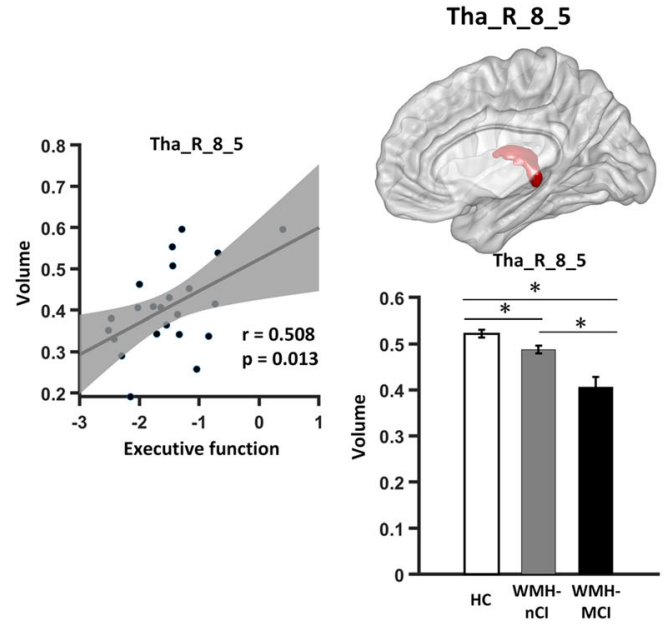

Fig. 3 Regions of significant correlation between mean grey matter volume and cognitive performance in WMH-MCI patients. Bar graphs: group differences in mean grey matter volume of the identified regions. Scatter plots: correlations between the mean grey

this study. In addition, the exploratory group classifications showed that regional GM measures can separate the WMHMCI and WMH-nCI groups with relatively high accuracy (77.3\% for cortical thickness; $83.3 \%$ for GMV; details in Tables S13, S14).

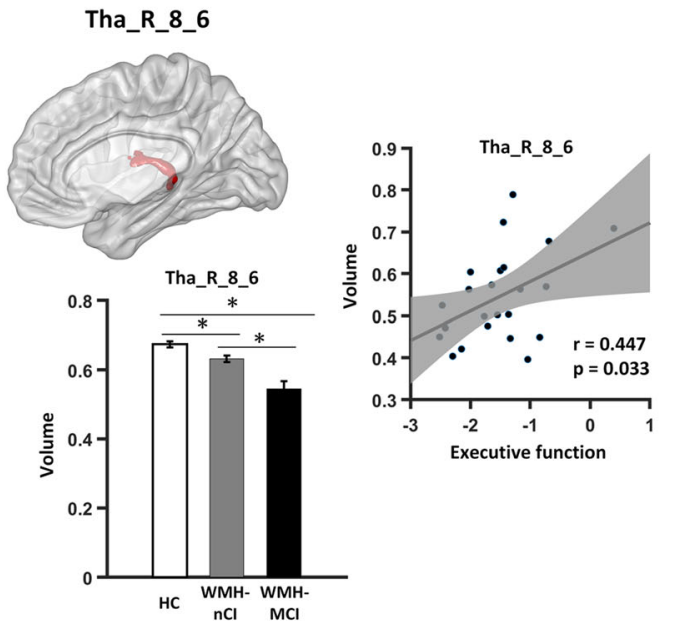

matter volume of identified regions and cognitive performance. IFG, inferior frontal gyrus; Tha, thalamus; $\mathrm{L}$, left; $\mathrm{R}$, right. $* P<0.05$ (Bonferroni corrected)

\section{Discussion}

In this work, we systemically investigated differences in whole-brain GM maps (GMV and cortical thickness) among moderate to severe WMH patients with and without 


\section{A Mediation effect of gray matter volume}

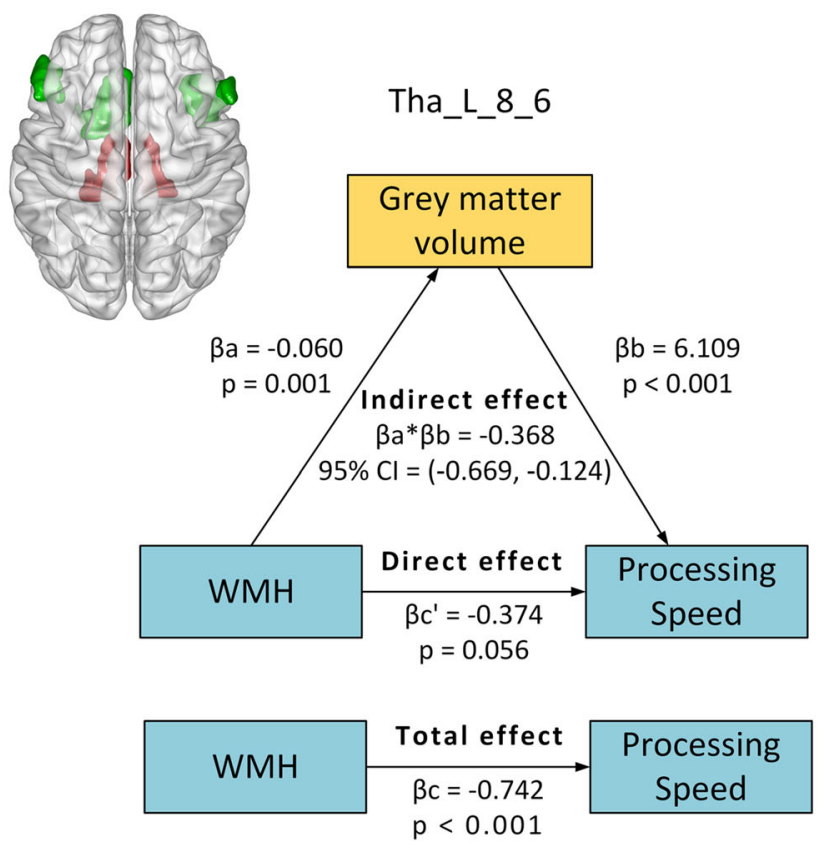

B Mediation effect of cortical thickness

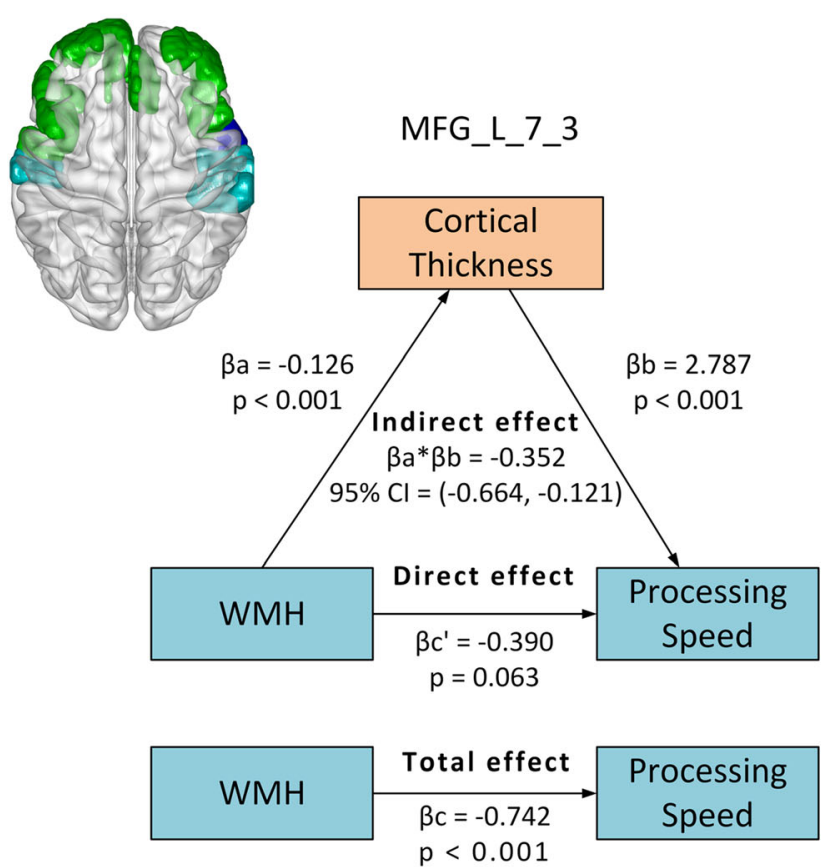

Fig. 4 Mediation effect of grey matter measures between WMH and cognitive function. Diagrams show areas of grey matter volume (A) or cortical thickness (B) mediating the relationship between $\mathrm{WMH}$ volume and cognitive performance (processing speed or executive function), and mediation models of the pathways from $\mathrm{WMH}$ volume to grey matter atrophy for grey matter volume $(\mathbf{A})$ and cortical thickness $(\mathbf{B})$ in representative regions (two subregions in the thalamus and frontal cortex) to reduce cognitive performance. In each model, age, sex, years of education, and TIV were entered as
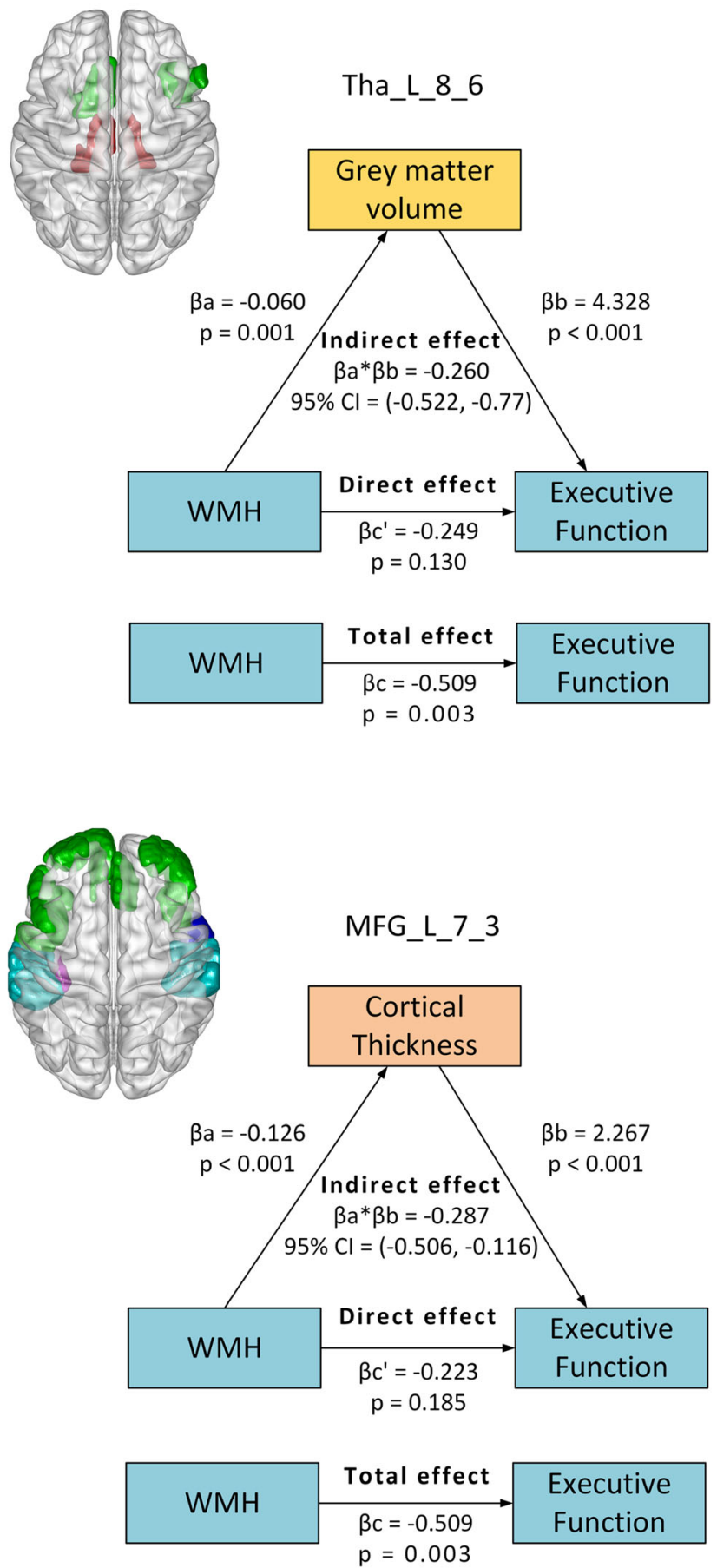

covariates. $\beta \mathrm{a}$ and $\beta \mathrm{b}$ represent the coefficients of the relationships between WMH volume and the regional grey matter measures (grey matter volume or cortical thickness), and the associations between the grey matter measures and cognition (when both WMH volume and grey matter measures were entered into the model as predicting variables). The mediating role of regional grey matter atrophy on the association between WMH and cognition is defined when the $95 \%$ confidence interval is entirely above or below 0 for 5,000 bootstrapping iterations. 
A

In-house

ADNI
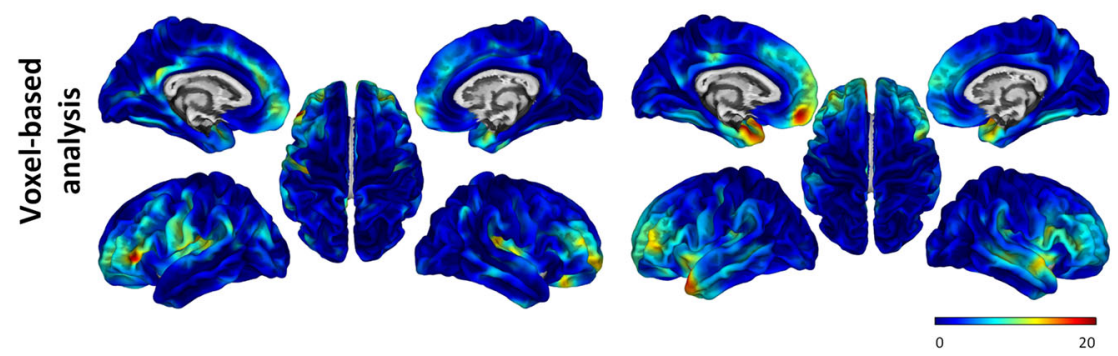

ADNI
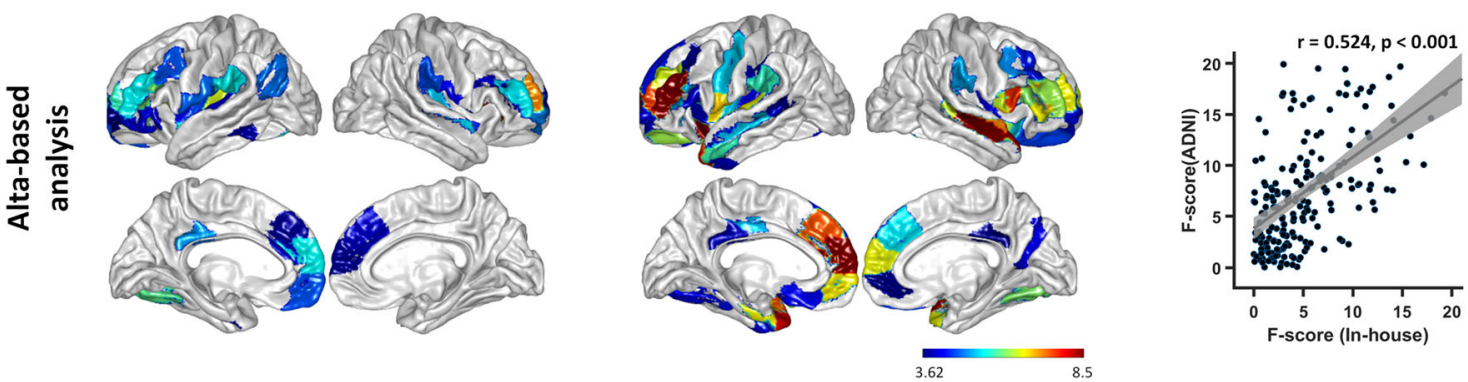

B
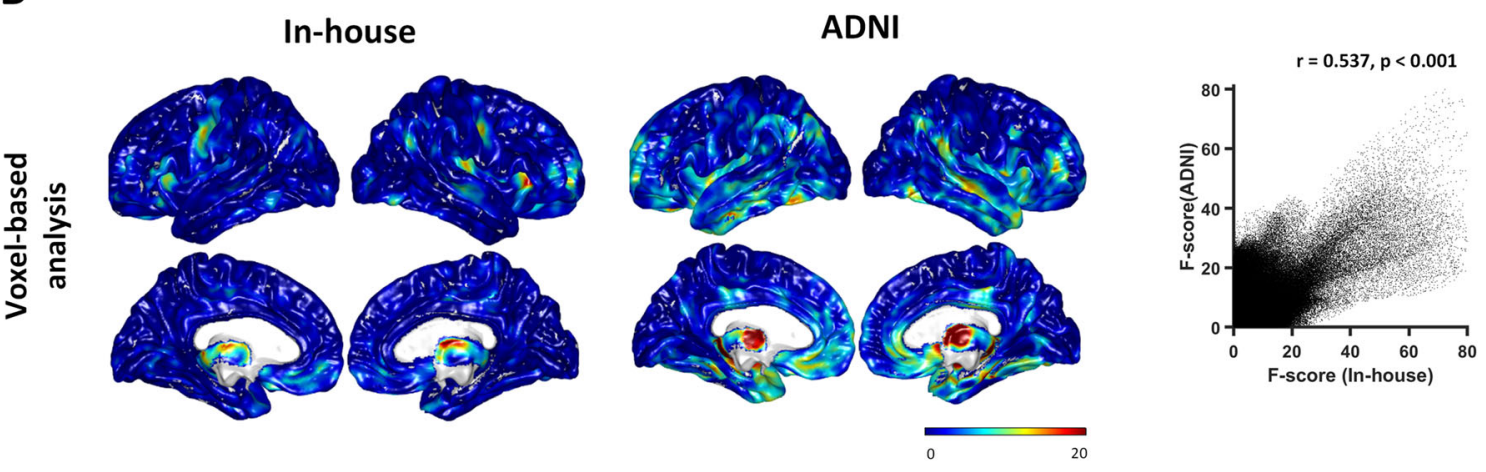

In-house

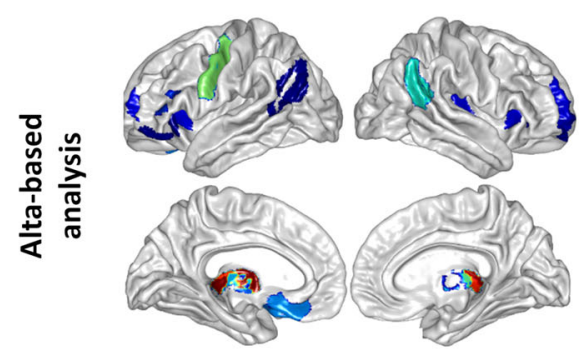

ADNI
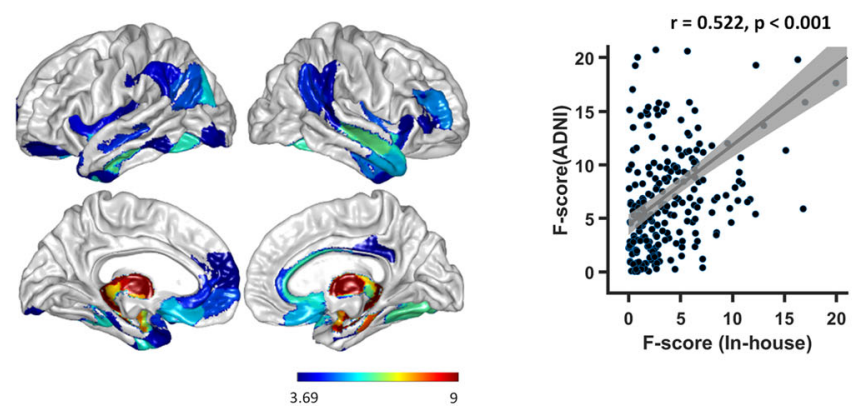

Fig. 5 Correlation analyses of the among-group differences in grey matter measures between the in-house dataset and the replication dataset from the ADNI database. Diagrams showing the statistical maps (left) and scatter plots (right) of correlation analyses between the $F$ scores of ANOVA for cortical thickness (A) and grey matter

volume (B) of the two independent datasets at the voxel and regional levels. The warmer and cooler colors indicate higher and lower correlation of grey matter alterations between the two datasets. ADNI, the Alzheimer's Disease Neuroimaging Initiative. 
MCI, and HCs at both the regional and the voxel level. Compared with HCs, both the WMH-MCI group and the WMH-nCI group showed a similar GM atrophy pattern in the bilateral thalamus, fronto-insular cortices, and a few parietal-temporal regions, and the pattern was more extensive and severe in the WMH-MCI than in the WMH-nCI group. Importantly, the GM-cognition correlation analyses and mediation analyses among GM, WMH, and cognition further identified the crucial role of specific GM atrophy in the thalamus and fronto-insular cortices underlying the early cognitive decline in WMH patients. Furthermore, the main results were convincingly well replicated in a series of control analyses including analyses at the voxel level, in an independent dataset from the ADNI database, and using different templates. In addition, exploratory classification analyses indicated that regional GM measures also distinguished WMH patients with and without early cognitive impairment. Altogether, these findings provide a novel insight into the mechanisms underlying WMH and subsequent cognitive decline.

Compared to HCs, both of the WMH groups exhibited volumetric loss of deep subcortical GM nuclei, especially the bilateral thalamus, in line with previous studies focused on subcortical vascular MCI patients [12] or patients with high WMH load from a community-dwelling cohort [43]. Furthermore, to extend their findings, we found that the WMH-MCI patients showed a more widespread and severe reduction of thalamic GMV than the WMH-nCI patients and further identified positive correlations between several subregions of the thalamus and cognition (processing speed and executive function) within the WMH-MCI group. Notably, our study for the first time revealed a mediating role of thalamic atrophy on the relationship between WMH load and cognitive decline in patients with WMH. Robust evidence has suggested that the thalamus works as a relay hub to the cerebral cortex and contributes to cognitive processing in diverse domains [44-46]. The characteristic atrophy of the thalamus underlying cognitive decline in WMH patients may be explained by two mechanisms. Primarily, as a subcortical manifestation that can be partly attributed to the chronic ischemia of CSVD, WMH not only reflects damage of the WM but also can invade subcortical GM nuclei directly [47]. The other explanation is that the thalamus has dense structural and functional connections to cortical regions, which are therefore vulnerable to hypometabolism and degeneration due to disruption of WM tracts even if there is no visible WMH in the thalamus on MRI [48-51]. In our previous studies, we have demonstrated functional thalamic disconnections in the cognitive decline of WMH patients using restingstate functional MRI [25]. And DTI studies have also shown that some projection fibers connecting the thalamus to cortical regions (i.e., the anterior thalamic radiation) are correlated with cognitive decline in CSVD patients with a high WMH load $[52,53]$. Given of the above, there is convergent evidence that the thalamus may be susceptible to WMH progression and damage of the thalamus develops along with the course of cognitive decline in WMH patients.

For the cerebral cortex, our results revealed shared and specific GM alterations in the WMH-MCI group relative to the WMH-nCI group and HCs; these mainly included reduced GMV and thinner cortical thickness in the frontoinsular cortices and a few regions of the parietal (mainly IPL) and temporal cortices. In particular, the cortical atrophy (mainly cortical thinning) in the majority of these regions was associated with cognitive decline in the WMHMCI group. Of note, previous studies have explored the pattern of cortical atrophy in patients with $\mathrm{WMH}$ and MCI [10-13]. Although it was hampered by the use of heterogeneous patient groups and methodological differences, our study, consistent with prior studies, showed that the reduction of cortical thickness and GMV in fronto-insular cortices was identified in patients with MCI and a high WMH load. The fronto-insular cortices, together with the thalamus, constitute the core regions of the salience network (SN), and are involved in a wide range of cognitive processes [54-56]. Meanwhile, the DLPFC and IPL, which are the hub of the executive control network (ECN) [56], were also identified as the characteristic atrophic regions in WMH-MCI patients compared with WMH-nCI patients and HCs. Playing essential and dissociable roles in human emotion and cognition [56, 57], the structural alterations of the SN and ECN coincide with the functional disconnection patterns underlying cognitive impairment in $\mathrm{WMH}$ patients revealed by previous studies $[25,58,59]$. Hence, we speculate that the selective GM atrophy of the hub regions in the SN and ECN may lead to functional abnormalities across the cognitive networks and this could be the crucial mechanism of WMH-related cognitive impairment.

One of the distinctive aspects in the present study was that we compared GM alterations among WMH patients with MCI, with those whose cognition was preserved and HCs. Thus, we obtained the characteristic GM alteration pattern in clinically early-stage WMH patients. Interestingly, compared with HCs, GM atrophy was also observed in the WMH-nCI patients, particularly in the thalamus and fronto-insular cortices noted above. In addition to being hub regions in cognitive networks, these regions that constitute the fronto-subcortical circuit are also thought to be involved in emotion and motor control. Thus, the specific GM atrophy of the thalamus and fronto-insular cortices in the WMH-nCI group corresponded to some extent to the disturbance of emotion and gait in WMH patients usually with an insidious onset that may occur 
before the cognitive impairment $[1,60]$. Another interesting finding is that increased GMV of the bilateral caudate was detected in both the WMH-MCI and WMH-nCI patients compared with $\mathrm{HCs}$, consistent with a previous study [11]. These novel findings may be a compensatory mechanism in the clinically early stage of WMH. Another possible explanation is that disturbance of the caudate may be responsible for the potential mood and motor disturbance in WMH patients, as revealed in 22q11.2 deletion syndrome [61] and vascular parkinsonism [62]. Although these findings were obtained at both the regional and the voxel level, and were repeated in the replication dataset from the ADNI database, further investigations is needed to verify the generalization and explore the mechanisms.

We further evaluated the relationships among GM alteration, $\mathrm{WMH}$, and cognition in $\mathrm{WMH}$ patients. To date, although some evidence has been obtained, the pathophysiology of cortical atrophy in WMH and subsequent cognitive impairment remains elusive. As noted in prior studies [16, 63], there are two possible mechanisms to explain this issue. First, considered to be the most important imaging manifestations and reflect the overall burden of CSVD, WMH may disturb WM fibers connecting cortical to subcortical GM, leading to secondary damage of the axonal cytoskeleton and cortical degeneration [64-66]. Our results add to the growing body of literature that implicates the specific disruption of WM tracts (i.e., the anterior thalamic radiation and corpus callosum) connected between subcortical and cortical regions in WMH patients [53, 65], which may cause a characteristic structurally and functionally aberrant frontosubcortical network and eventually lead to cognitive impairment. The other possible mechanism is that direct damage by CSVD, such as cortical microinfarcts and microbleeds, may also be responsible for cortical atrophy in $\mathrm{WMH}$ and the subsequent cognitive impairment. Nevertheless, it is difficult to explain the characteristic damage pattern in WMH patients with this hypothesis and further investigation is needed in studies with susceptibilityweighted images, 3D FLAIR, or double inversion recovery sequences $[67,68]$.

We also performed a series of control analyses (repeated the between-group comparisons at the voxel level, in an independent dataset from the ADNI database, using multiple measures and different atlases, and further controlling for risk factors such as hypertension and diabetes) to verify the reproducibility and generalization of the main results of GM alterations in WMH patients with and without cognitive impairment. Notably, whether revealed in another independent dataset or using different templates, the GM alterations across the three groups exhibited very similar distribution patterns, highlighting the important role of the thalamus and fronto-insular cortices in WMH progression and subsequent cognitive impairment. Furthermore, since different sensitivity in capturing different types of structural changes and pathological processes $[23,69,70]$, the similar GM alteration patterns evaluated among the three groups by using different measures (GMV $v s$ cortical thickness, and atlas-based $v s$ voxel-based approaches), were able to demonstrate the reproducibility and generalization of our main results from multiple perspectives, although there were great disparities in age, race, and ethnicity, as well as MRI scanners between two datasets which may lead to differences of altered regions across the three groups revealed by the two datasets. Besides, the results of exploratory classification analyses indicated that regional GM measures may serve as a novel and potential marker for detecting the early cognitive decline in patients with a high WMH load at the individual level.

Despite these advances, this study had several limitations that should be addressed. First, the sample size of the in-house dataset was relatively small, particularly for the WMH-MCI group, and the sizes of the WMH-MCI and WMH-nCI groups were unbalanced due to the study design, which may lead to lower statistical power. Based on studies focused on the neuroimaging features of $\mathrm{AD}$ [71, 72], the characteristic GM alteration pattern in WMH patients with MCI needs further cross-validation by largescale multi-center studies in the future. Second, we did not conduct a comprehensive evaluation of motor and mood disturbances which are common in patients with WMH. Hence, an influence of these factors on the results cannot be completely excluded. Finally, since various types of pathology (cerebral ischemia, aging, and AD-related degeneration) may participate in the mechanisms of WMH progression and cognitive impairment in WMH patients [73], longitudinal studies with $\mathrm{A} \beta$ and tau measures are warranted, to reach further consensus on specific GM alterations underlying cognitive decline in WMH and evaluate the clinical value of GM for the prediction of early cognitive impairment in WMH patients.

\section{Conclusion}

Here, we demonstrated the characteristic structural GM alteration pattern in WMH patients with and without MCI, and further indicated that specific GM atrophy in the thalamus and fronto-insular cortices underlie the cognitive decline in $\mathrm{WMH}$ patients. Importantly, the alteration pattern in the main results was verified in a series of control analyses, highlighting the robustness and generalizability of our data. Our results provide novel insights into the mechanisms of WMH progression and the subsequent cognitive impairment in patients with a high WMH load. 
Acknowledgements We are grateful to Kun Zhao for technical assistance, and Prof. Min Zhang and Prof. Daishi Tian for recruiting the participants. This work was supported by the National Natural Science Foundation of China (81801146, 81871438, and 81771341), the Natural Science Foundation of Hubei Province (2017CFB392), Beijing Natural Science Funds for Distinguished Young Scholars (JQ200036), and the Flagship Program of Tongji Hospital, China (2019CR106). Data collection and sharing for this project was funded by the Alzheimer's Disease Neuroimaging Initiative (ADNI) (National Institutes of Health Grant U01 AG024904) and DOD ADNI (Department of Defense Award number W81XWH-12-2-0012). The ADNI was funded by the National Institute on Aging, the National Institute of Biomedical Imaging and Bioengineering, and generous contributions from AbbVie, Alzheimer's Association; Alzheimer's Drug Discovery Foundation; Araclon Biotech; BioClinica, Inc.; Biogen; Bristol-Myers Squibb Company; CereSpir, Inc.; Cogstate; Eisai Inc.; Elan Pharmaceuticals, Inc.; Eli Lilly and Company; EuroImmun; F. Hoffmann-La Roche Ltd, and its affiliated company Genentech, Inc.; Fujirebio; GE Healthcare; IXICO Ltd.; Janssen Alzheimer Immunotherapy Research \& Development, LLC.; Johnson \& Johnson Pharmaceutical Research \& Development LLC.; Lumosity; Lundbeck; Merck \& Co., Inc.; Meso Scale Diagnostics, LLC.; NeuroRx Research; Neurotrack Technologies; Novartis Pharmaceuticals Corporation; Pfizer Inc.; Piramal Imaging; Servier; Takeda Pharmaceutical Company; and Transition Therapeutics. The Canadian Institutes of Health Research provided funds to support ADNI clinical sites in Canada. Private sector contributions were facilitated by the Foundation for the National Institutes of Health (www.fnih. org). The grantee organization was the Northern California Institute for Research and Education, and the study was coordinated by the Alzheimer's Therapeutic Research Institute at the University of Southern California. ADNI data were disseminated by the Laboratory for Neuro Imaging at the University of Southern California.

Conflict of interest All authors declare that they have no conflict of interest.

Open Access This article is licensed under a Creative Commons Attribution 4.0 International License, which permits use, sharing, adaptation, distribution and reproduction in any medium or format, as long as you give appropriate credit to the original author(s) and the source, provide a link to the Creative Commons licence, and indicate if changes were made. The images or other third party material in this article are included in the article's Creative Commons licence, unless indicated otherwise in a credit line to the material. If material is not included in the article's Creative Commons licence and your intended use is not permitted by statutory regulation or exceeds the permitted use, you will need to obtain permission directly from the copyright holder. To view a copy of this licence, visit http://creativecommons. org/licenses/by/4.0/.

\section{References}

1. Prins ND, Scheltens P. White matter hyperintensities, cognitive impairment and dementia: an update. Nat Rev Neurol 2015, 11 : 157-165.

2. Debette S, Markus HS. The clinical importance of white matter hyperintensities on brain magnetic resonance imaging: systematic review and meta-analysis. BMJ 2010, 341: c3666.

3. Moonen JE, Foster-Dingley JC, van den Berg-Huijsmans AA, de Ruijter W, de Craen AJ, van der Grond J. Influence of small vessel disease and microstructural integrity on neurocognitive functioning in older individuals: the DANTE study leiden. AJNR Am J Neuroradiol 2017, 38: 25-30.

4. Lawrence AJ, Chung AW, Morris RG, Markus HS, Barrick TR. Structural network efficiency is associated with cognitive impairment in small-vessel disease. Neurology 2014, 83: 304-311.

5. van Leijsen EMC, Bergkamp MI, van Uden IWM, Ghafoorian M, van der Holst HM, Norris DG, et al. Progression of white matter hyperintensities preceded by heterogeneous decline of microstructural integrity. Stroke 2018, 49: 1386-1393.

6. Peres R, De Guio F, Chabriat H, Jouvent E. Alterations of the cerebral cortex in sporadic small vessel disease: A systematic review of in vivo MRI data. J Cereb Blood Flow Metab 2016, 36: 681-695.

7. Liu C, Li C, Gui L, Zhao L, Evans AC, Xie B, et al. The pattern of brain gray matter impairments in patients with subcortical vascular dementia. J Neurol Sci 2014, 341: 110-118.

8. Li C, Du H, Zheng J, Wang J. A voxel-based morphometric analysis of cerebral gray matter in subcortical ischemic vascular dementia patients and normal aged controls. Int J Med Sci 2011, 8: 482-486.

9. Mueller SG, Mack WJ, Mungas D, Kramer JH, CardenasNicolson $\mathrm{V}$, Lavretsky $\mathrm{H}$, et al. Influences of lobar gray matter and white matter lesion load on cognition and mood. Psychiatry Res 2010, 181: 90-96.

10. Seo SW, Ahn J, Yoon U, Im K, Lee JM, Tae Kim S, et al. Cortical thinning in vascular mild cognitive impairment and vascular dementia of subcortical type. J Neuroimaging 2010, 20: $37-45$.

11. Wang J, Liang Y, Chen H, Wang W, Wang Y, Liang Y, et al. Structural changes in white matter lesion patients and their correlation with cognitive impairment. Neuropsychiatr Dis Treat 2019, 15: 1355-1363.

12. Yi L, Wang J, Jia L, Zhao Z, Lu J, Li K, et al. Structural and functional changes in subcortical vascular mild cognitive impairment: a combined voxel-based morphometry and resting-state fMRI study. PLoS One 2012, 7: e44758.

13. Liu C, Zhao L, Yang S, Luo Y, Zhu W, Zhu W, et al. Structural changes in the lobar regions of brain in cerebral small-vessel disease patients with and without cognitive impairment: An MRIbased study with automated brain volumetry. Eur J Radiol 2020, 126: 108967.

14. Knopman DS, Griswold ME, Lirette ST, Gottesman RF, Kantarci $\mathrm{K}$, Sharrett AR, et al. Vascular imaging abnormalities and cognition: mediation by cortical volume in nondemented individuals: atherosclerosis risk in communities-neurocognitive study. Stroke 2015, 46: 433-440.

15. Swardfager W, Cogo-Moreira $\mathrm{H}$, Masellis $\mathrm{M}$, Ramirez J, Herrmann N, Edwards JD, et al. The effect of white matter hyperintensities on verbal memory: Mediation by temporal lobe atrophy. Neurology 2018, 90: e673-e682.

16. Tuladhar AM, Reid AT, Shumskaya E, de Laat KF, van Norden AG, van Dijk EJ, et al. Relationship between white matter hyperintensities, cortical thickness, and cognition. Stroke 2015, 46: 425-432.

17. Rizvi B, Narkhede A, Last BS, Budge M, Tosto G, Manly JJ, et al. The effect of white matter hyperintensities on cognition is mediated by cortical atrophy. Neurobiol Aging 2018, 64: 25-32.

18. Besteher B, Squarcina L, Spalthoff R, Bellani M, Gaser C, Brambilla $\mathrm{P}$, et al. Brain structural correlates of irritability: Findings in a large healthy cohort. Hum Brain Mapp 2017, 38 : 6230-6238.

19. Ediri Arachchi W, Peng Y, Zhang X, Qin W, Zhuo C, Yu C, et al. A systematic characterization of structural brain changes in schizophrenia. Neurosci Bull 2020, 36: 1107-1122. 
20. Hutton C, Draganski B, Ashburner J, Weiskopf N. A comparison between voxel-based cortical thickness and voxel-based morphometry in normal aging. Neuroimage 2009, 48: 371-380.

21. Panizzon MS, Fennema-Notestine C, Eyler LT, Jernigan TL, Prom-Wormley E, Neale M, et al. Distinct genetic influences on cortical surface area and cortical thickness. Cereb Cortex 2009, 19: 2728-2735.

22. Skup M, Zhu H, Wang Y, Giovanello KS, Lin JA, Shen D, et al. Sex differences in grey matter atrophy patterns among AD and aMCI patients: results from ADNI. Neuroimage 2011, 56: 890-906.

23. Hopkins WD, Li X, Crow T, Roberts N. Vertex- and atlas-based comparisons in measures of cortical thickness, gyrification and white matter volume between humans and chimpanzees. Brain Struct Funct 2017, 222: 229-245.

24. Fan L, Li H, Zhuo J, Zhang Y, Wang J, Chen L, et al. The human brainnetome atlas: a new brain atlas based on connectional architecture. Cereb Cortex 2016, 26: 3508-3526.

25. Zhu W, Huang $\mathrm{H}$, Yang S, Luo X, Zhu W, Xu S, et al. Dysfunctional architecture underlies white matter hyperintensities with and without cognitive impairment. J Alzheimers Dis 2019, 71: 461-476.

26. Fazekas F, Chawluk JB, Alavi A, Hurtig HI, Zimmerman RA. MR signal abnormalities at $1.5 \mathrm{~T}$ in Alzheimer's dementia and normal aging. AJR Am J Roentgenol 1987, 149: 351-356.

27. Helenius J, Henninger N. Leukoaraiosis burden significantly modulates the association between infarct volume and national institutes of health stroke scale in ischemic stroke. Stroke 2015, 46: $1857-1863$.

28. Mirza SS, Ikram MA, Bos D, Mihaescu R, Hofman A, Tiemeier H. Mild cognitive impairment and risk of depression and anxiety: A population-based study. Alzheimers Dement 2017, 13: 130-139.

29. Brissos S, Dias VV, Soeiro-de-Souza MG, Balanza-Martinez V, Kapczinski F. The impact of a history of psychotic symptoms on cognitive function in euthymic bipolar patients: a comparison with schizophrenic patients and healthy controls. Braz J Psychiatry 2011, 33: 353-361.

30. Peterson KA, Savulich G, Jackson D, Killikelly C, Pickard JD, Sahakian BJ. The effect of shunt surgery on neuropsychological performance in normal pressure hydrocephalus: a systematic review and meta-analysis. J Neurol 2016, 263: 1669-1677.

31. Jia J, Zhou A, Wei C, Jia X, Wang F, Li F, et al. The prevalence of mild cognitive impairment and its etiological subtypes in elderly Chinese. Alzheimers Dement 2014, 10: 439-447.

32. Jia J, Wang F, Wei C, Zhou A, Jia X, Li F, et al. The prevalence of dementia in urban and rural areas of China. Alzheimers Dement 2014, 10: 1-9.

33. Dahnke R, Yotter RA, Gaser C. Cortical thickness and central surface estimation. Neuroimage 2013, 65: 336-348.

34. Spalthoff R, Gaser C, Nenadic I. Altered gyrification in schizophrenia and its relation to other morphometric markers. Schizophr Res 2018, 202: 195-202.

35. Besteher B, Gaser C, Ivansic D, Guntinas-Lichius O, Dobel C, Nenadic I. Chronic tinnitus and the limbic system: Reappraising brain structural effects of distress and affective symptoms. Neuroimage Clin 2019, 24: 101976.

36. Pell GS, Briellmann RS, Chan CH, Pardoe H, Abbott DF, Jackson GD. Selection of the control group for VBM analysis: influence of covariates, matching and sample size. Neuroimage 2008, 41: 1324-1335.

37. Malone IB, Leung KK, Clegg S, Barnes J, Whitwell JL, Ashburner $\mathrm{J}$, et al. Accurate automatic estimation of total intracranial volume: a nuisance variable with less nuisance. Neuroimage 2015, 104: 366-372.
38. Hayes AF. Introduction to Mediation, Moderation, and Conditional Process Analysis: A regression-based approach. The Guilford Press 2013.

39. Jack CR Jr, Bernstein MA, Fox NC, Thompson P, Alexander G, Harvey $\mathrm{D}$, et al. The Alzheimer's Disease Neuroimaging Initiative (ADNI): MRI methods. J Magn Reson Imaging 2008, 27: 685-691.

40. Carmichael O, Xie J, Fletcher E, Singh B, DeCarli C, Alzheimer's Disease Neuroimaging I. Localized hippocampus measures are associated with Alzheimer pathology and cognition independent of total hippocampal volume. Neurobiol Aging 2012, 33: 1124 e1131-1141.

41. Glasser MF, Coalson TS, Robinson EC, Hacker CD, Harwell J, Yacoub E, et al. A multi-modal parcellation of human cerebral cortex. Nature 2016, 536: 171-178.

42. Urchs S, Armoza J, Moreau C, Benhajali Y, St-Aubin J, Orban P, et al. MIST: A multi-resolution parcellation of functional brain networks. MNI Open Res 2019, 1.

43. Su N, Liang X, Zhai FF, Zhou LX, Ni J, Yao M, et al. The consequence of cerebral small vessel disease: Linking brain atrophy to motor impairment in the elderly. Hum Brain Mapp 2018, 39: 4452-4461.

44. Hwang K, Bertolero MA, Liu WB, D’Esposito M. The human thalamus is an integrative hub for functional brain networks. J Neurosci 2017, 37: 5594-5607.

45. Mitchell AS, Sherman SM, Sommer MA, Mair RG, Vertes RP, Chudasama Y. Advances in understanding mechanisms of thalamic relays in cognition and behavior. J Neurosci 2014, 34: 15340-15346.

46. Bell PT, Shine JM. Subcortical contributions to large-scale network communication. Neurosci Biobehav Rev 2016, 71: 313-322.

47. Jacqmin M, Herve D, Viswanathan A, Guichard JP, During M, Dichgans $\mathrm{M}$, et al. Confluent thalamic hyperintensities in CADASIL. Cerebrovasc Dis 2010, 30: 308-313.

48. Bjorklund E, Lindberg E, Rundgren M, Cronberg T, Friberg H, Englund E. Ischaemic brain damage after cardiac arrest and induced hypothermia-a systematic description of selective eosinophilic neuronal death. A neuropathologic study of 23 patients. Resuscitation 2014, 85: 527-532.

49. Houtchens MK, Benedict RH, Killiany R, Sharma J, Jaisani Z, Singh $\mathrm{B}$, et al. Thalamic atrophy and cognition in multiple sclerosis. Neurology 2007, 69: 1213-1223.

50. Pulsinelli WA, Brierley JB, Plum F. Temporal profile of neuronal damage in a model of transient forebrain ischemia. Ann Neurol 1982, 11: 491-498.

51. Metzger CD, van der Werf YD, Walter M. Functional mapping of thalamic nuclei and their integration into cortico-striatal-thalamocortical loops via ultra-high resolution imaging-from animal anatomy to in vivo imaging in humans. Front Neurosci 2013, 7: 24.

52. Duering M, Gesierich B, Seiler S, Pirpamer L, Gonik M, Hofer E, et al. Strategic white matter tracts for processing speed deficits in age-related small vessel disease. Neurology 2014, 82: 1946-1950.

53. Duering M, Zieren N, Herve D, Jouvent E, Reyes S, Peters N, et al. Strategic role of frontal white matter tracts in vascular cognitive impairment: a voxel-based lesion-symptom mapping study in CADASIL. Brain 2011, 134: 2366-2375.

54. Fair DA, Dosenbach NU, Church JA, Cohen AL, Brahmbhatt S, Miezin FM, et al. Development of distinct control networks through segregation and integration. Proc Natl Acad Sci U S A 2007, 104: 13507-13512.

55. Dosenbach NU, Fair DA, Miezin FM, Cohen AL, Wenger KK, Dosenbach RA, et al. Distinct brain networks for adaptive and 
stable task control in humans. Proc Natl Acad Sci U S A 2007, 104: 11073-11078.

56. Seeley WW, Menon V, Schatzberg AF, Keller J, Glover GH, Kenna $\mathrm{H}$, et al. Dissociable intrinsic connectivity networks for salience processing and executive control. J Neurosci 2007, 27: 2349-2356.

57. Yi LY, Liang X, Liu DM, Sun B, Ying S, Yang DB, et al. Disrupted topological organization of resting-state functional brain network in subcortical vascular mild cognitive impairment. CNS Neurosci Ther 2015, 21: 846-854.

58. Dey AK, Stamenova V, Turner G, Black SE, Levine B. Pathoconnectomics of cognitive impairment in small vessel disease: A systematic review. Alzheimers Dement 2016, 12: 831-845.

59. Qin Q, Tang Y, Dou X, Qu Y, Xing Y, Yang J, et al. Default mode network integrity changes contribute to cognitive deficits in subcortical vascular cognitive impairment, no dementia. Brain Imaging Behav 2021, 15: 255-265.

60. Group LS. 2001-2011: a decade of the LADIS (Leukoaraiosis And DISability) Study: what have we learned about white matter changes and small-vessel disease? Cerebrovasc Dis 2011, 32: 577-588.

61. Ching CRK, Gutman BA, Sun D, Villalon Reina J, Ragothaman $\mathrm{A}$, Isaev $\mathrm{D}$, et al. Mapping subcortical brain alterations in 22q11.2 deletion syndrome: effects of deletion size and convergence with idiopathic neuropsychiatric illness. Am J Psychiatry 2020, 177: 589-600.

62. Dunet V, Deverdun J, Charroud C, Le Bars E, Molino F, Menjot de Champfleur S, et al. MRI volumetric morphometry in vascular parkinsonism. J Neurol 2017, 264: 1511-1519.

63. Mok V, Wong KK, Xiong Y, Wong A, Schmidt R, Chu W, et al. Cortical and frontal atrophy are associated with cognitive impairment in age-related confluent white-matter lesion. J Neurol Neurosurg Psychiatry 2011, 82: 52-57.

64. Du AT, Schuff N, Chao LL, Kornak J, Ezekiel F, Jagust WJ, et al. White matter lesions are associated with cortical atrophy more than entorhinal and hippocampal atrophy. Neurobiol Aging 2005, 26: 553-559.

65. Glickstein M, Berlucchi G. Classical disconnection studies of the corpus callosum. Cortex 2008, 44: 914-927.

66. Jones DK, Lythgoe D, Horsfield MA, Simmons A, Williams SC, Markus HS. Characterization of white matter damage in ischemic leukoaraiosis with diffusion tensor MRI. Stroke 1999, 30: 393-397.

67. Wardlaw JM, Smith EE, Biessels GJ, Cordonnier C, Fazekas F, Frayne R, et al. Neuroimaging standards for research into small vessel disease and its contribution to ageing and neurodegeneration. Lancet Neurol 2013, 12: 822-838.

68. Ter Telgte A, van Leijsen EMC, Wiegertjes K, Klijn CJM, Tuladhar AM, de Leeuw FE. Cerebral small vessel disease: from a focal to a global perspective. Nat Rev Neurol 2018, 14: 387-398.

69. Xiao Y, Lui S, Deng W, Yao L, Zhang W, Li S, et al. Altered cortical thickness related to clinical severity but not the untreated disease duration in schizophrenia. Schizophr Bull 2015, 41: 201-210.

70. Anticevic A, Dierker DL, Gillespie SK, Repovs G, Csernansky JG, Van Essen DC, et al. Comparing surface-based and volumebased analyses of functional neuroimaging data in patients with schizophrenia. Neuroimage 2008, 41: 835-848.

71. Jin D, Zhou B, Han Y, Ren J, Han T, Liu B, et al. Generalizable, reproducible, and neuroscientifically interpretable imaging biomarkers for Alzheimer's disease. Adv Sci (Weinh) 2020, 7: 2000675

72. Zhao K, Ding YH, Han Y, Fan Y, Alexander-Bloch AF, Han T, et al. Independent and reproducible hippocampal radiomic biomarkers for multisite Alzheimer's disease: diagnosis, longitudinal progress and biological basis. Sci Bull 2020, 65: 1103-1113.

73. Jonkman LE, Kenkhuis B, Geurts JJG, van de Berg WDJ. Postmortem MRI and histopathology in neurologic disease: a translational approach. Neurosci Bull 2019, 35: 229-243. 